\title{
HISTORIA CONSTITUCIONAL DEL ELEGTOR MEXICANO 1812-1994
}

\author{
Dr. Juan de Dios Gonzálfz Ibarra*
}

\section{ANTECEDENTES}

A partir de la definición del clector, como el sujeto social ${ }^{1}$ que elige a sus representantes pudiendo controlar sus acciones por medio de elecciones competitivas, y un conjunto de normas jurídicas que permiten ese control de los gobernantes, y el poder exigirles explicaciones de sus atctos y proyectos a desarrollar en todo momento, estando el poder delegado en diversos órganos, existiendo políticos de oficio o representantes profesionales por la complejidad de la vida moderna que encuentra en el disenso y en el pluralismo político una forma de la realidad que en lugar de reprimirse, como se hacia anteriormente, sc consideran elementos valiosos de la realidad social siempre y cuando no dañen la existencia de otros valores y prácticas políticas, realizaremos este estudio.

La historia del elector mexicano transcurre a partir del antecedente de la Constitución de Cádiz o Constitución Política de la Monarquía Española, promulgada en esa ciudad de la entonces metrópoli el 19 de marzo de 1812, en la cual se establece que "las cortes son la reunión cle todos los diputados que representan la nación, nombrados por los ciudadanos en la forma que dirá". ${ }^{2}$

El artículo 91 definió que para poder ser diputado de cortes se requería ser ciudadano que estuviera en el ejercicio de sus derechos, mayor de veinticinco años, y que haya nacido en la provincia o esté avecindado en ella con residencia a lo menos de siete años, bien sea del estado seglar o del eclesiástico secular; pudiendo recaer la clección en

- Profesor de Política Tributaria en la División de Estudios de Posgrado en la lacultad de Derecho de la UNAM.

1 Zamelman, H. y Valencia, G., Los sujetos sociales una propuesta de análisis, ch Revista Acta Sociológica, vol. II, núm. 2, UNAM, FCPS, mayo-agosto de 1990, p. 89.

2 García Orozoo, A., Legislación electoral mexicana 1812-1977, Comisión Federal Electoral, México, 1978, p. 1. 
los ciudadanos que componen la junta, o en los de fuera de ella ${ }^{3}$ se exigía además ser propietario de una renta anual que se originara en bienes propiedad del candidato, conforme lo que acordarían las cortes en cuanto a la calidad de los bienes y el monto de la renta.

El Decreto Constitucional para la libertad de la América Mexicana o Decreto Constitucional de Apatzingán del 22 de octubre de 1814, estableció que se requería para ejercer el cargo de diputado "ser ciudadano en ejercicio de sus derechos, contar con treinta años de edad, buena reputación, patriotismo acreditado con servicios positivos y tener luces no vulgares para desempeñar las augustas funciones de este empleo","4 se perdía la calidad de ciudadano por crimen de herejía, apostatía y traición a la nación.

Como podemos deducir el componente religioso sigue presente en la primera Constitución mexicana, en relación con la calidad de ciudadano.

Por el lado del representante diputado señaló que debía tener pleno ejercicio como ciudadano en sus derechos, 30 años de edad, buen prestigio, patriota conforme hechos demostrados y educación idónea para poder ejercer las actividades que como diputado le serían encomendadas.

Se estableció la prohibición de que no podían ser al mismo tiempo diputados dos o más parientes en segundo grado, así como la no relación inmediata.

Los electores elegirán por cada parroquia un elector parroquial, quienes a su vez, elegirán a un elector de partido para integrar las juntas electorales de partido.

Estos últimos electores conforme el artículo 93 integrarán "respectivamente, las juntas provinciales, que para nombrar los diputados deben incorporarse en el Congreso, se han de celebrar en cada provincia o en el pueblo que señalare el intendente, a quien toca presidirlos, y fijar el día, hora y sitio en que hayan de verificarse". 5

Los electores de partido formarían las juntas provisionales, las que nombrarán a los diputados que constituirán el congreso supremo, compuesto por un diputado elegido por cada provincia con autoridad igual de cada uno de ellos, éstos durarán en su encargo no más de dos años. En cuanto a los tres individuos que forman el Supremo Gobierno,

3 Op. cit., p. 8.

4 Decreto constitucional para la libertad de la América mexicana, artículo 52. 22-X-1814, Imprenta Nacional, en Tena Ramirez, F., Leyes Fundamentales de Mexico, 1808-1987, Editorial Porrúa, México, 1987, p. 37.

s op. cit., p. 42. 
"El Supremo Gobierno los elegirá en sesión secreta, hecha la elección, continuará la sesión en público, y el secretario anunciará al pueblo las personas que hubieran resultado elegidas, durando en el cargo cuatro años".

El Supremo Tribunal de Justicia se compondrá de cinco individuos, iguales en autoridad, y que fungirán por el lapso de tres años.

Finalmente conforme el artículo 232 estableció que el Supremo Gobierno formará el plan conveniente para convocar a la representación nacional, bajo la base de la población y con arreglo a los demás principios del derecho público.

\section{LA CONSTITUCION DE 1824}

Esta fue la primera que tuvo fuerza legal en nuestro pais, recientemente independizado de España y al respecto estableció que la Cámara de Diputados se compondría de representantes elegidos en su totalidad cada dos años, esto conforme al artículo octavo.

De acuerdo al modelo federal sostenido por esta Constitución, con Ramos Arizpe como presidente de la comisión redactora, que tuvo vigencia hasta su abrogación en 1835 , las cualidades determinadas para poder ser elector se dejaban a cargo de lo que establecieran las legislaturas de los estados, "federalismo viene del concepto de federación, y éste a su vez del vocablo latino foedus o federare, que nos expresa ligar, unir o alianza, por lo que consecuente un Estado Federal será aquel que fue formado por varios estados o cntidades que permanecían separados antes del pacto"; " para Carpizo en un Estado Federal constitucionalmente existen dos órdenes coordinados entre sí: el de la federación y el de las entidades federativas, gozando éstas de sus propias constituciones y autonomía, interviniendo en el proceso de reforma de la Constitución General de la Nación. ${ }^{7}$

Siguiendo con esta forma de organización sociopolítica el Poder Legislativo Federal se constituyó, por vez primera, con un Congreso General compuesto por una Cámara de Diputados y otra de Senadores, cada una de esas asambleas legislativas tenían diferentes atribuciones; se concede en teoría que los diputados representan a los habitantes en general y los senadores a los habitantes de esas entidades.

6 Gamiz Parral, M., Derecho Constiucional y Administrativo de las Entidades Federativas, UNAM, Méxiro, 1990, p. 39.

7 Op. cit., p. 40, Jorge Carpizo, citado en esta obra, es de los constitucionalistas con más prestigio en el país y cn Latinoamérica. 
Se adoptó la forma de República representativa popular federal, teniendo a la religión católica como la oficial y prohibió cualquier otra, con Estados independientes, libres y soberanos, con la división de poderes de legtislativo, ejecutivo y judicial.

La calidad de ciudadano al dejarse a los estados se definió regionalmente y, por lo que respecta al bicamerismo "en Veracruz en 1825, en Durango en 1826 y en Yucatán en 1825 se dieron los únicos casos de bicamerismo local, debido a la creación de un Senado estatal en cada una de esas entidades federativas, durante un periodo demasiado corto". ${ }^{8}$

El municipio libre con antecedentes en el siglo XIX en la Constitución de Cádiz que estableció a los ayuntamientos integrados por alcaldes, regidores, síndico con el jefe político a la cabeza y, en su defecto, por el alcalde para el gobierno directo de los pueblos (artículo 309); en la Constitución de 1824, entendemos que confiere a las entidades federativas la mejor forma de organizarse, pues no son mencionados en ella.

El ayuntamiento tiene su origen etimológico en el latín municipium que descompuesto en sus componentes muni y civitas significa un trabajo de la ciudad, en la Nueva España Hernán Cortés, el 22 de abril de 1519, fundó el primer ayuntamiento de estas tierras en la Villa Rica de la Vera Cruz para así legalizar sus acciones. ${ }^{9}$

De acuerdo con esta Constitución, para ser diputado se requiere tener 25 años, dos años de vecindad en el Estado a que se aspire a ser elegido o haber nacido en él, no pueden serlo los que estén privados o suspensos de los derechos de ciudadano, el presidente y el vicepresidente, los ministros de la Suprema Corte de Justicia, los secretarios, los gobernadores, arzobispos y obispos.

La Cámara de Diputados se compondrá de representantes elegidos cada dos años en su totalidad, por los ciudadanos de los estados.

EI Senado se compondrá de dos senadores por cada estado, elegidos a mayoría absoluta de votos por sus legislaturas y renovados por unidad cada año, debiendo contar con 30 años de edad.

Para ser presidente o vicepresidente es necesario ser ciudadano mexicano por nacimiento, contar con 35 años y ser residente del país.

Las legislaturas de cada estado elegirán por mayoría absoluta de votos, a dos individuos, el que reúna la mayorfa absoluta de los votos de las legislaturas será el presidente y el vicepresidente lo sería el que

8 Ibidem, p. 267.

- Véase p. 317. 
siguiera en votos, según los artículos 84 y 85 del título IV sección 1a., durarían cuatro años en sus cargos.

\section{BASES Y LEYES CONSTITUCIONALES DE LA REPÚBLICA} MEXICANA DE 1836

Al triunfo del grupo conservador encabezado por Antonio López de Santa Anna y Lucas Alamán, entre otros, y con la derrota de los liberales con líderes como Gómez Farías, Josć Luis Mora, Benito Juárez y Melchor Ocampo, se promulgaron las llamadas Siete Leyes o Constitución Centralista que en relación con el equilibrio de poderes adoptó un elemento novedoso consistente en su artículo 4o. Instituye que "se establecerá un árbitro suficiente para que ninguno de los tres (poderes) pueda traspasar los límites de sus atribuciones, éste es el Supremo Poder Conservador, formado por cinco miembros, elegido por las juntas departamentales", teniendo como requisitos que fueran mexicanos por nacimiento, estar en ejercicio de sus derechos ciudadanos, 40 años, renta anual de tres mil pesos y haber desempeñado ciertos cargos, esto conforme la segunda ley, éste es un antecedente de la Comisión de los Derechos Humanos y existe en varios países. ${ }^{10}$

La ley tercera estableció que para ser diputado se requería "ser mexicano por nacimiento o natural de cualquier parte de la América que en 1810 dependía de la España, y sea independiente, si se hallaba en la República al tiempo de su emancipación", contar con 30 años de edad ya cumplidos el día electoral, con pleno ejercicio de sus derechos como ciudadano, vecino u originario del departamento al que fuera a representar, tener un ingreso anual de cuando menos mil quinientos pesos con base en su capital.

Los senadores, que suman veinticuatro, requerían haber cumplido el día de la elección 35 años, en pleno ejercicio de sus derechos ciudadanos, con ingresos anuales mínimos de 2,500 pesos, los elegiría el Supremo Poder Conservador, tomando en cuenta las propuestas de la Cámara de Diputados, el gobierno en junta de ministros y la Suprema Corte de Justicia.

El senado debía renovarse por terceras partes cada dos años. El mismo procedimiento para elegir senadores se aplicará para la elección del

10 Tena Ramírez, F., Leyes Fundamentales de México 1808-1987, Editorial Porrúa, México, 1987, p. 210 
presidente, con un periodo de ocho años, siendo nombrado por el congreso, en Iugar del Supremo Poder Conservador.

De acuerdo al proyecto centralista la República se organizaría en departamento, distritos y partidos, los departamentos estarían a cargo de gobernadores, en los distritos prefectos designados por el gobernador, con la ratificación del gobierno general y en los partidos subprefectos.

En las capitales de los departamentos se integrarían ayuntamientos elegidos popularmente, siendo dirigidos por alcaldes, regidores y síndicos.

Al no señalarse explícitamente la calidad de ciudadano, se podía inducir de los requisitos establecidos para ser elegidos representantes.

\section{LA CONSTITUCION POLITICA DE LA REPÚBLICA MEXICANA DE 1842}

Con esta Constitución vuelven al escenario político dominante los liberales después del periodo centralista del 36, sin embargo, la fuerza de los partidarios del centralismo se mantuvo por algún tiempo, encabezada por el general Antonio López de Santa Anna en pugna con esta Constitución.

Aquí nos apoyaremos en el segundo proyecto del 3 de noviembre de 1842. La calidad de ciudadano se adquiría a los 18 años siendo casado y 21 si no lo era, con empleo y forma honesta de vivir, se estipula también ${ }^{11}$ con el artículo 7o., que a partir de 1850 debería saber leer y escribir.

Se establece el derecho de votar y ser votado y poder ejercer cualquier empleo público.

Los ciudadanos mexicanos ejercerían el poder electoral en las elecciones primarias, por cada 200 habitantes se nombraria un elector secundario, quien tendría que saber leer y escribir, con 25 años de edad y con los requisitos que señalaran sus respectivos departamentos.

Los electores secundarios, conforme el artículo 18, integran las juntas secundarias.

Se deja de contemplar la necesidad de poseer cierto patrimonio para poder ser elector, sólo el leer y escribir en los electores secundarios los que representaban como dijimos a cada 200 electores.

11 Op. cit., p. 373. 
Las garantías individuals figuran en este documento político a partir de la igualdad, libertad, seguridad y propiedad.

Se sigue considerando a la religión católica, apostólica y romana como la oficial, sin tolerarse públicamente el ejercicio de otra.

Se hace la distinción entre poder público general y departamental.

Para poder ser diputado era necesario ser originario o avecindado cn el lugar de elección con antigüedad de cuatro años cuando menos, tener veinticinco años cumplidos y un capital "físico o moral" (artículo 35) que produjera una renta anual no menor de mil doscientos pesos, sin embargo, de este último requisito se dispensaba a los profesores de establecimientos públicos que hubieran ejercido la docencia durante cinco años.

Los departamentos nombrarían un diputado por cada 70,000 almas o fracción que rebasara la mitad.

Conforme a la organización departamental se elegirían dos senadores, quienes requerían lo mismo que los aspirantes a diputados, pero con 35 años de edad y renta de tres mil pesos.

Los diputados en su totalidad se cambiarían cada dos años y los senadores por mitad cada bienio.

Para ser presidente se requería la edad de 40 años, pertenecer al estado secular y no haber sido condenado a pena corporal legalmente.

El colegio departamental elegiría al presidente.

Los departamentos tendrían sus respectivas asambleas, gobernadores y tribunales.

CONSTITUCION FEDERAL DE LOS ESTADOS UNIDOS MEXICANOS DEL 5 DE FEBRERO DE 1857

Su antecedente es el movimiento del Plan de Ayutla de 1854 junto con el desprestigio de Antonio López de Santa Anna y el resurgimiento de los liberales encabezados por Benito Juárez, Melchor Ocampo, Ponciano Arriaga y un componente moderado representado por Ignario Comonfort, Manuel Doblado, Lafragua.

La calidad de ciudadano se adquiere a los 18 años si se es casado o veintiuno si no lo fuera, poseyendo una forma honesta de vida.

Se contempla por primera vez en nuestra vida constitucional, dentro de los derechos de los ciudadanos, el poder "asociarse para tratar los asuntos políticos del país" (artículo 35 fracción III), o sea que las organizaciones políticas se reconocen como parte de la realidad del juego político nacional. 
También se menciona lo ya contemplado en otras constitucioncs como son el votar, ser votado y nombrado para cualquier puesto público, conforme los requisitos de ley.

Dentro de los cambios nos encontramos con la obligación ciudadana de "inscribirse en el padrón de su municipalidad, manifestando la propiedad que tiene, o la industria, profesión o trabajando de que subsiste". 12

La religión finalmente desaparece del plano político-constitucional.

Los requisitos para ser diputado son que sea ciudadano en ejercicio de sus derechos, con 25 años de edad, vecino del estado o territorio que lo elija y no pertenecer al estado eclesiástico.

Se redujo el número de electores de 70,000 a 40,000 o fracción que superara la mitad.

Los diputados debían ser electos de manera indirecta en un primer momento, en votación secreta, conforme lo que señalara -otra innovación - una ley electoral.

La calificación de las elecciones quedó a cargo de la cámara respectiva del congreso.

Cabe señalar que la elección del Presidente de los Estados Unidos Mexicanos era indirecta también "en primer grado y en escrutinio secreto", ${ }^{13}$ y remitía a una ley electoral, duraba cuatro años en el cargo, contando con 35 años cumplidos, mexicanos por nacimiento.

La soberanía nacional se atribuye al pueblo, así se parte de considerar que original y por esencia proviene de él, el poder público también surge por y para su beneficio (artículo 39). ${ }^{14}$

La soberanía se ejerce a través de los poderes federales y estatales.

Se establece la federación de estados libres y soberanos en lo interior, que se integran en una república representativa, democrática, federal bajo los principios de esta Constitución y las estatales que no pueden contravenir a aquella.

CONSTITUCION POLITICA DE LOS ESTADOS UNIDOS MEXICANOS QUE REFORMA LA DEL 5 DE FEBRERO DE 1857

Al triunfo de la revolución de 1910, varias corrientes políticas se manifestaron en el Congreso Constituyente de Querétaro, encabezadas

12 Ibidem, p. 612.

13 Véase el articulo 76.

14 Op. cit., p. 613 . 
una por el Primer Jefe del Ejército Constitucionalista don Venustiano Carranza, otra por el general Alvaro Obregón Salido y la que contaba con el general Francisco J. Mújica como representante más connotado.

El mismo título de esta Constitución, señala la necesidad de conciliar a la corriente encabezada por Carranza que deseaba el amplio respecto de la Constitución anterior, con algunas modificaciones, y aquella que planteaba otro modelo de organización sociocconómica para el país.

Las discusiones sobre el artículo 27 y 129 constitucionales, reflejan en plenitud la profundidad de la materia de estos artículos, que transformaban radicalmente las posibilidades de organización de la nueva sociedad, que se había empezado a gestar desde las entrañas del porfiriato para parirse con la sangre de la revolución triunfante.

La calidad de ciudadano se adquiere a los 18 años si se es casado o 21 si no lo es, y conforme una manera honesta de vivir.

Son derechos y obligaciones políticas del ciudadano votar, ser votaclo y nombrado para empleo o comisión pública, conforme cumpla los requisitos del cargo, y asociarse políticamente para intervenir en los asuntos de esta índole, inscribirse en el padrón electoral.

El poder legislativo sigue depositándose en el Congreso Gencral, com sus cámaras de diputados y senadores.

Diputados, representante de la nación, debía ser mexicano por nacimiento, con 25 años de edad, con pleno ejercicio de sus derechos ciudadanos, haber nacido en la entidad federativa o territorio o vecino de éste con más de 6 meses de permanencia, aparte de otras prohibiciones se establece que no podrá ser "ministro de algún culto religioso". 15

Se disponía en el artículo 51 originalmente que el diputado duraría en su encargo dos años, a partir de 1933 esto se reformó para quedar en tres años y se prohibió la reelección inmediata.

Por cada 170,000 habitantes o fracción superior a los ochenta mil, se elegirá un diputado, con su respectivo suplente, sin que ninguna entidad federativa pueda tener menos de dos diputados propietarios.

El candidato a senador deberá cumplir los mismos requisitos del diputado, salvo la edad que se eleva a 35 años, en un principio duraban 4 años, Io que se elevó a 6 , debiendo renovarse por mitades éste.

16 Ibidem, p. 612 . 
Las elecciones son directas y cada cámara realiza la calificación de las elecciones de sus miembros.

Para ser presidente se requiere contar con 35 años de edad, ser ciudadano mexicano, elevándose el requisito de nacimiento al establecerse que deberá ser hijo de padres mexicanos por nacimiento, duraba 4 años en su cargo, esto se modificó en 1934 fijándose en 6 , sin posibilidad de reelección, siendo por voto directo su elección, al igual que los otros representantes.

Haciendo un análisis conforme las últimas reformas constitucionales relativas promulgadas el 5 de abril de 1990, así como las anteriores tenemos que actualmente la edad para ser elector es de 18 años sea hombre o mujer, la Cámara de Diputados se integra por 300 diputados electos según el principio de mayoría relativa y 200 de representación proporcional, la de senadores se mantuvo con 64 miembros, renovándose el $50 \%$ cada trienio.

EI sistema electoral mexicano ha experimentado grandes cambios de 1812 a 1991, que han hecho que se identificara como "una formación clásica del liberalismo: predominio de liderazgos personales que organizaban clubes o partidos de estabilidad precaria sin organización para instruir programas permanentes; limitaciones en cuanto a requisitos económicos y de propiedad para alcanzar la calidad de elector o funcionario electoral evitando, con ello, la incorporación masiva, amén de la incapacidad electoral de la mujer; votación indirecta en segundo o tercer grado; voto público que no contempla la separación entre el registro de electores y la boleta electoral y elección de mayoría simple" (sin embargo por las reformas de 1993-1994 esto ya no es aplicable en la actualidad).

La Revolución mexicana quebró, en su centro, ese régimen cerrado y desigual para recuperar otro en letra y espíritu que reconocía al pueblo como depositario de la soberanía, y la libre participación del individuo en igualdad de derechos y garantías como su vía regia de expresión. ${ }^{16}$

Con base en esta posición Rebolledo Gout sostenia que "derrotada militarmente la dictadura y elegido Madero como presidente de la República, el Gobierno se propuso recuperar el sentido primario de la función del sistema electoral: fijar el mecanismo de expresión de la soberanía popular a los representantes legítimamente electos. Para ello

15 Varios, México 75 Años de Revolución III. Desarrollo Político. Molinar, J., El Sistema Electoral, Fondo de Cultura Económica, México, 1988, p. 388. 
promovió, congruentemente, modificaciones al marco electoral en $19 \mathrm{I}$, introduciendo, entre otras, el sufragio individual y secreto y la votación por medio de boletas individuales y distintas a las listas electorales". ${ }^{77}$

Luis Medina consideraba que "puede afirmarse que en el México independiente del siglo XIX, los avances de la materia son bien escasos". Los vaivenes de las luchas civil s, que en el fondo no expresan más que la anemia de la sociedad civil, llevaron a los liberales a instaurar un sistema de elección indirecto altamente descentralizado. Si bien es cierto que en la Constitución y en Ia Ley Orgánica Electoral de 1857 se introducen algunos conceptos avanzados como son el distrito electoral y el voto universal, el reformismo liberal en la materia quedó trunco. En las discusiones del Congreso Constituyente, en donde se enfrentaron puros y moderados, predominó el criterio de estos últimos: la ignorancia generalizada del pueblo obliga a la cautela en Ia implantación del voto directo y, si bien se rechazó la elección en tres escalones, se adoptó la indirecta en primer grado. Aún más, el manejo del proceso electoral -determinación de los distritos electorales, elaboración del padrón, instalación e integración de casillas- quedó en manos de gobernadores, jefes políticos y los ayuntamientos. ${ }^{18}$

Este autor sostiene que lo difícil de la entonces situación nacional, caracterizada por la lucha entre los poderes centrales y locales -éstos representados por caciques y caudillos locales y regionales-, se impuso pragmáticamente al proyecto de una verdadera federación, esto traevá como consecuencia el que el sistema electoral no registre avances hasta después de la caída del porfiriato, en que se confía en la idea de que el voto en las manos del pueblo conduce hacia mejores soluciones sociales.

Lo anterior se confirma dos veces: con la inmediata publicación de la Ley Electoral del 19 de diciembre de 1911, por parte de Francisco Indalecio Madero en que establecía el voto secreto e individual, conservándose todavía la limitación del analfabetismo como obstáculo para tener la calidad de votante y elecciones primarias y secundarias. Con las reformas de 22 de mayo de 1912 a Ias Leyes Electorales, decretadas por el mismo Madero, se establecieron las elecciones directas de diputados y senadores.

77 Op. cit., p. 388.

18 Medina, Luis, Ia Evolución Electoral en el México Contemporáneo, Comisión Federal Electoral, México, 1978, p. 8. Esta obra junto con la de Juan Rebolledo Gout, son fundamentales para entender la dinámica del sistema clectoral mexicano. 
Carranza al triunfo del movimiento constitucionalista, expide la Ley Electoral para la formación del Congreso Constituyente el 20 de septiembre de 1916, con lo que define reglas abiertas para integrar ese cuerpo legislativo, dándole de principio una base política formal de sustento a la nueva Constitución.

Es tal su interés y reconocimiento a la cuestión electoral que a unas horas de promulgada la Constitución y conforme al artículo 2o. transitorio decreta la Ley Electoral del 6 de febrero de 1917 en la que "se apoya al pueblo mexicano a elecciones extraordinarias de Presidente de la República, Diputados y Senadores al Congreso de la Unión".19

Con posterioridad, el 2 de julio de 1918, ya en el cargo de Presidente Constitucional Carranza, el Congreso decreta la Ley para la elección de poderes federales que con distintas reformas de 1920, 1921, 1931, 1942 y 1943 reguló los procesos electorales del país hasta la Ley Electoral Federal de 1946.20

Medina establece Ia siguiente periodización: "A raíz de la revolución mexicana de 1910 , se puede considerar que el sistema electoral mexicano ha pasado por una serie de etapas, cuyas constantes serían tres: la centralización como el medio para garantizar mejor el desarrollo del acto electoral; el propósito de encuadrar las corrientes de opinión política en organismos nacionales, permanentes y estables, y por último, la búsqueda de un medio que, sin alterar sustancialmente el régimen de gobierno estatuido en la Constitución, permita la participación en las tareas legislativas federales y estatales de los partidos minoritarios. Este proceso, que cubre un periodo que se extiende de 1911 a la actualidad, es producto de experiencias electorales concretas, de altibajos políticos, incluso de irrupciones violentas.

Muchos han sido los obstáculos y problemas a los que se ha buscado dar respuesta haciendo adecuaciones y reformas dentro de las tres. líneas arriba mencionadas; también ha habido errores, que tarde o temprano han dado lugar a una abundante cosecha de desconfianza".21

19 García OROzco, A., Legislación Electoral Mexicana, 1912-1977, Comisión Federal Electoral, México, 1978, p. 255.

20 PaOLI Bolio, Francisco J., Legislación Electoral y Proceso Politico 1917-1982, en las Elecciones en México: evolución y perspectivas, Siglo XXI Editores, México, 1985, P. 130. La afirmación que hace este intelectual, en torno a la influencia de esta ley en las siguientes es interesante.

21 Op. cit., p. 131. 
Por su parte, Núñez ${ }^{22}$ afirma que nuestro país pasa por un momento de cambio hacia un nivel más elevado en la democracia.

Junto a las voces que sostienen que se ha presentado un gran avance en materia electoral, ${ }^{23}$ se presentaban los de la oposición que, como en el caso de Michoacán en 1992, consideran que no es confiable el padrón electoral y se cuestiona la transparencia del voto.

El avance en este ámbito implica no sólo lo formal-jurídico, sino la práctica que es la que define básicamente a la realidad electoral.

A partir de las reformas constitucionales del 2 de septiembre de 1993 se modificaron siete artículos constitucionales que son el 4, 54, 56, 60, 63,74 y 100 , que junto con el decreto del lo. de julio de 1994, fracción I, rclativa a que a partir del 31 de diciembre de 1999 podrá ser presidente el "ciudadano mexicano por nacimiento, en pleno goce de sus derechos, hijo de padre o madre mexicanos y haber residido en el país al nacer durante veinte años","*4 nos presentaron aspectos innovadores en relación con la historia constitucional del elector mexicano, que dan una nueva personalidad a éste desde la creación del organismo autónomo ${ }^{25}$ con personalidad y patriotismo propio llevado al Tribunal Fedcral Electoral, suprema autoridad jurisdiccional electoral (también recordemos que el Instituto Federal Electoral no existía en 1988, con su órgano supremo el Consejo General "ciudadanizado", o tal vez, con más precisión convendría identificarla como "electorizado" para diferenciarla cle la dirección desde arriba o estatal).

Los diputados, conforme la suma resultante de la representación proporcional y pluricional, de un solo partido político no serán más de 3I5, por su parte cl número de senadores se elevó de dos a cuatro por cada entidad federativa, tres electos por mayoría relativa y uno correspondicnte al partido que resulte con el segundo lugar en votos, o sea, la primera ninoría.

El principio de autocalificación del poder legislativo pasó a la historia y, hoy la Cámara de Diputados se clige en colegio electoral, sólo

\$2 Núñfz Jiménez, A., El suevo sistema electoral mexicano, Fondo de Cultura Económica, México, 1991, p. 13.

23 NÚÑEZ JiMÉNFZ, A., op. cit., p. 34.

24 Diario Oficial, 1 de julio de 1994, México.

25 Diario Oficial de la Federación, 3 de septiembre de 1993, México. Tomado de la Crónica de la Reforma Política Federal, Instituto de Investigaciones Legislativas, México, 1994. 
para calificar la elección del Presidente de los Estados Unidos Mexicanos. ${ }^{26}$

La votación del $77.74 \%$ es un dato verificable que muestra que las reformas constitucionales del COFIPE y los pactos de civilidad formadas por todos los partidos políticos le dieron confianza al elector respecto a que su voto sería rigurosamente defendido y válido, así en las 96 mil $411^{27}$ casillas de los 300 distritos electorales, conforme la votación válida que es aquella que de la votación total se le restan los votos nulos ${ }^{28}$ integrada por 34 millones 549 mil 501 sufragios válidos, ${ }^{29}$ el $50.18 \%$ favoreció al candidato del Partido Revolucionario Institucional para Presidente de la República Ernesto Zedillo con 17 millones. 336 mil 325 votos; Diego Fernández de Ceballos consiguió el 26.69\%, o sea, 9 millones 222 mil 899 votos y Cuauhtémoc Cárdenas obtuvo 5 millones 901 mil 557 votos, los que representan el $17.08 \%$ de la votación válida presidencial. ${ }^{30}$

Los cambios registrados en la Constitución, los resultados de 1994 y los pactos de civilidad nos impulsaron a continuar con el estudio del elector mexicano, ciertos de que el sistema electoral se mejorará conforme las experiencias electorales, que el abstencionismo y el millón 782 de votos anulados equivalentes al $2.28 \%$ indican que el camino es largo, que zún debemos luchar por un México más justo social, económica y políticamente reflexionando, sin embargo, entendamos que la. transparencia, legalidad, credibilidad y legitimidad avanzaron -con alguna falta, como en las casillas especiales- para bien de todos nosotros.

\footnotetext{
26 Op. cit., p. 424.

27 Periódico Reforma, año 1, núm. 269, domingo 28 de agosto de 1994.

28 Periódico La Jornada, año 9, núm. 3581, domingo 28 de agosto de 1994 .

29 Reforma, op. cit.

so Reforma y La Jornada de la fecha citada.
} 